\title{
Buckling analysis of thin-walled sigma beams with respect to different numerical descriptions
}

\author{
K. Rzeszut \\ Institute of Building Engineering, Poznan University of Technology, Poznań, Poland \\ I. Szewczak \\ Faculty of Civil Engineering and Architecture, Lublin University of Technology, Lublin, Poland \\ P. Różyło \\ Faculty of Mechanical Engineering, Lublin University of Technology, Lublin, Poland
}

M.J. Guminiak

Institute of Structural Analysis, Poznan University of Technology, Poznań, Poland

\begin{abstract}
The main aim of the study is buckling analysis of steel beams made of thinwalled cold-formed sigma profile with respect different numerical descriptions. The analyses are carried out on sigma profile with a height of $140 \mathrm{~mm}$ and a span of $2.20 \mathrm{~m}$. The numerical models of the Finite Element Method (FEM), developed in the Abaqus program, include modelling of the so-called boundary conditions of the forks with use of displacement limitations. The beams are modelled using S4R shell finite element with linear or square shape function. Local and global instability behaviour is investigated using linear buckling analysis and are verified by the comparisons with theoretical critical bending moment obtained from analytical close form formulas based on so called Vlasow beam theory dedicated to the thinwalled elements. In addition, the engineering analysis of buckling is carried out for a simple shell (plate) model of the separated cross-section part in form of flange wall using Boundary Element Method (BEM). The discussion concerning geometric simplification in sigma crosssection according theoretical assumptions is performed too. It is worth noting that the value of the critical bending moment calculated on the basis of the Vlasov beam theory does not take into account the loss of local stability or contour deformation. On the other numerical shell FEM models enable multimodal buckling analysis taking into account interactive buckling. In the paper eigenvalue and shape of buckling modes for selected numerical models are calculated for three first buckling modes but the values of critical bending moments are identified basing on the eigenvalue obtained for the first buckling mode.
\end{abstract}

\section{INTRODUCTION}

The history of research on the stability of structures is almost 250 years old and it was started in 1759 by Euler, who published a work on buckling in compression columns. Euler's work was continued by, inter alia, Timoshenko and Vlasov, who formulated a general theory of calculating thin-walled bars in a complex state of stress and elastic loss of stability. The theoretical and experimental studies on the stability of thin-walled elements have shown the shortcomings of classical linear theories and revealed the need for more advanced analyses. It became possible thanks to the development of computer methods and the formulation of a geometric matrix and

DOI: $10.1201 / 9781003132134-20$ 
incremental equations, and later the use of the Finite Element method has been comprehensively described by Zienkiewicz (1977). Loss of stability is the most important phenomenon that causes the load capacity of thin-walled metal structures to be exceeded. For this reason, stability conditions are the subject of a large part of design standards and have been introduced into the curriculum in engineering, master's and doctoral programs. In engineering practice two classes of stability problems of cold-formed members, have been distinguished. The first of them, the global stability is analysed according to Vlasov's theory, which takes into account torsion of the bar, assuming a non-deformable contour. The second class concerns the theory of post-critical load capacity taking into account the local stability of walls. When assessing the bearing capacity of building structures, it may be dangerous to consider these two classes of problems separately. Therefore, when assessing the load-bearing capacity of the elements according to Vlasov's theory, the influence of wall stability should be additionally investigated in accordance with the plate theory so called effective length concept proposed by Karman (1932). Many software for computer aided design of metal structures contain ready-made procedures for taking into account the influence of instability problems on the structure capacity. However, the phenomenon of stability is so complex that a routine approach to the design of thin-walled structures, as proposed by professional computer programs, can be dangerous or can lead to uneconomical projects. More over the problem becomes more complicated when there is a need to define the critical moment for symmetrical sections, where the axis of symmetry is the weaker one, e.g. channels or sigma sections Rzeszut (2018). The Polish standard PN-90/B-03200 provides a simplified method of checking the bearing capacity and torsion of beams made of C-section. In the case of no interaction of the bending moment and the sheer force, it allowed to determine the approximate share of torsion in the stress state of the channel section at approx. 15\%. On the other hand, the PN-EN 1993-1-1 Standard does not provide any method of determining the critical moment for symmetrical sections, where the axis of symmetry is the weaker axis, and refers the designer to basic knowledge on the strength of materials. This work involved the reliability assessment of the results of calculations of critical moments of cold-rolled sigma cross-section using analytical formulas based on the Vlasov beam theory, contained in AISI (1996), Bródka (2006) and Szczerba (2013) and Finite Element Method (FEM) using the Abaqus program and solid or shell finite elements. In addition, the paper attempts to analyse stability using Boundary Element Method (BEM) for the separate wall of profile.

\section{NUMERICAL ANALYSIS}

\subsection{Analytical formulas}

The critical bending moment can be determined based on the analytical procedures contained in standards such as PN-90/B-03200 or PN-EN 1993-1-1 but as it was mentioned above, in case of sections with only one weaker axis of symmetry the critical moment can be calculated using formula contained in AISI (1996):

$$
\mathrm{M}_{\mathrm{cr}}=\frac{C_{b} \pi^{2} E I_{y}}{l_{e}^{2}} \sqrt{\frac{I_{\omega}}{I_{y}}}
$$

where: $l_{e}$ - o lateral-torsional buckling length, $I_{y}$ - second moment of area with respect to the main axis of the section, perpendicular to the bending direction, $I_{\omega}-$ warping constant, $C_{b}-$ coefficient corresponding to bending moment variation along the bar length.

\subsection{Finite element method}

The problem of finding a solution to the eigenvalue equation, i.e. Eigenvalue problem often appears in linear buckling issue (also called as Eigenvalue buckling). This analysis predicts the theoretical buckling strength of an ideal elastic structure in form of critical load. In FEM 
analysis the eigenvectors $U$ have been computed by solving the linear eigenvalue problem which can be described as follow:

$$
\left(\mathbf{K}^{\mathrm{o}}+\lambda \mathbf{K}^{\mathrm{G}}\right) \mathbf{U}=0
$$

where $\lambda$ is the load multiplier and eigenvector $\mathbf{U}$ represents the buckling mode shapes, $\mathbf{K}^{\mathrm{O}}$ is the linear stiffness matrix, $\mathbf{K}^{\mathrm{G}}$ is the initial geometric matrix. In Eq. (2) the proportional loading and linearization of the pre-buckling state was assumed. The critical buckling loads are $\lambda_{i}{ }_{i} \mathbf{P}$, where $\mathbf{P}$ is the reference load (the base state).

\subsection{Boundary element method}

The initial stability problem of the plate structures can also be solved in an engineering manner using the Boundary Element Method (BEM). The complex steel profile can be divided into simple elements - plates, fixed at the edges. It is also assumed, that the considered plate is subjected to in-plane loading, which can have the constant or linear character along single plate edge.

The thin plate bending is described by the differential equation:

$$
D \nabla^{4} w=-\bar{p}
$$

where $D=E h^{3} /\left(12\left(1-v^{2}\right)\right)$ is the plate stiffness and

$$
\bar{p}=N_{x} \frac{\partial^{2} w}{\partial x^{2}}+2 N_{x y} \frac{\partial^{2} w}{\partial x \partial y}+N_{y} \frac{\partial^{2} w}{\partial y^{2}}
$$

is the substitute loading.

The solution of the equation (3) can be expressed as the integral representation in which the appropriate boundary values take place: the shear force, the bending moment, deflection, the angle of rotation in normal direction and the angle of rotation in tangent direction which is not independent and is calculated using a plate boundary deflection. Presented approach proposed by Guminiak (e.g. 2014, 2016) avoids Kirchhoff forces at the plate corner and equivalent shear forces at a plate boundary wherein two unknown variables are still considered at the boundary element node. The set of internal collocation points is introduced to establish a plate curvatures (Guminiak 2014, 2016). Alternatively the governing integral equations are derived using Betti's theorem. The solution of differential equation (4) in form of integral representation has the form of boundary-domain integral equations. Finally, after elimination of boundary variables the standard eigenvalue problem can be obtained (Guminiak 2014, 2016). The number of boundary elements is 160 and the number of internal collocation points is 320 .

\section{NUMERICAL EXCAMPLES}

The numerical analysis were carried out on sigma thin-walled beam made by "Blachy Pruszyński". The dimensions of cross-section of sigma profile was as follow: height $-140 \mathrm{~mm}$, the flange width $-70 \mathrm{~mm}$ and a wall thickness $-2.5 \mathrm{~mm}$. All tested beams were simply supported elements with the span of $2.20 \mathrm{~m}$ and loaded by uniformly distributed actions. A detailed description of laboratory tests and laboratory stand scheme are described in the paper (Rzeszut 2017, 2018). Beams were made of S350 GD steel grade, characterized by Young modulus E=201.8 $\mathrm{GPa}$, Poisson ratio $v=0.282$ and Yield stresses $\mathrm{f}_{\mathrm{y}}=418.5 \mathrm{MPa}$ determined on the basis of own material coupon test. The developed numerical models were different in terms of boundary conditions and, the type of finite element (FE). Two different types of finite elements were used, namely S4R type with a linear shape function (four-node type with reduced integration) (FEM- 
shell-1), and a shell element of S8R type with a square shape function was used (FEM-shell-s). The size of finite elements is $7.5 \mathrm{~mm}$. The supports were modelled in order to reflect so-called fork support. The supports were modelled using the displacement constraints imposed on partition plane of the beam in supports zone in distance of $0.4 \mathrm{~m}$ from the beam end in form of constrained horizontal displacements $\mathrm{Ux}$ at the web and edge stiffener, and in form of constrained vertical displacements Uy on the bottom flange. At all points located on this partition plane rotation was free (Figure 1). In order to meet Vlasov theory assumptions, among the others, that the resultant load should pass through the shear centre of the cross-section several numerical models were developed (see Figure 2). Various methods of external load modelling are marked with symbols V1, V2, V3. In the case of the V1 and V2, the uniformly distributed load was applied to the theoretical line located at the level of the upper flange of the cross-section and located directly above the shear centre of the cross-section. In the case V1, a reference point has been assigned to a non-deformable "WIRE line" (as part of 3D/Discrete Rigid modelling), which in turn determines the boundary conditions with the load as the unit force. Throughout the analysis, the line was a non-deformable part and was directly coupled to the entire cross-section of the Sigma type member. In the case V2, a non-deformable line of the WIRE type was assigned a reference point, in which the boundary conditions with the load being the unit force were determined. Throughout the analysis, the line was a non-deformable part and was directly coupled to the separated area (a line of a length adequate to the distance between the supports) located in the middle of the cross-section height of the Sigma type structure. In the case V3, uniformly distributed unit load was applied over the entire width of the upper flange and along the length equal to the spacing of supports. The methods of applying the external load in each case are shown in Figure 2.

a)

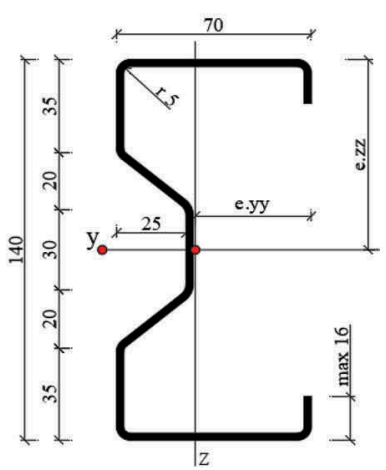

b)

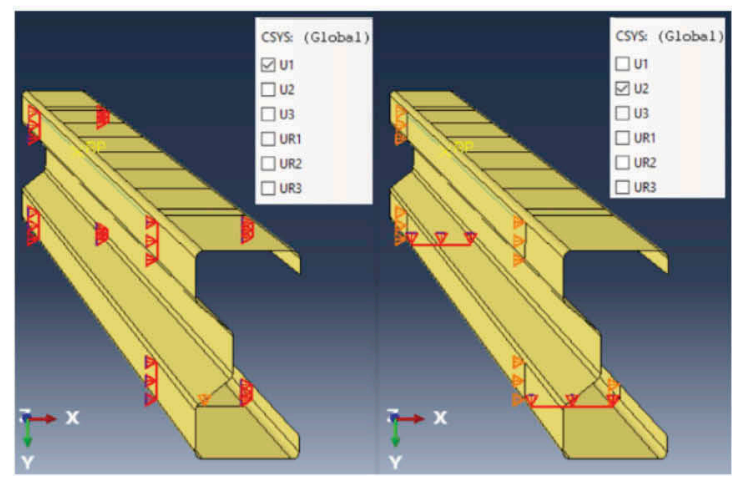

Figure 1. Numerical model. a) cross-section geometry $\Sigma 140 \times 70 \times 2.5$, b) support - displacement constraints.

a)

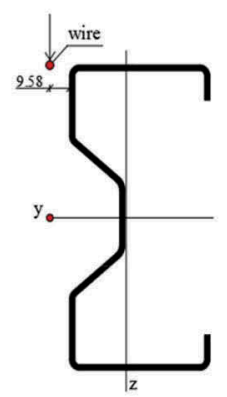

b)

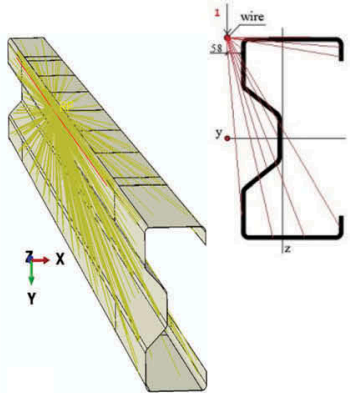

c)

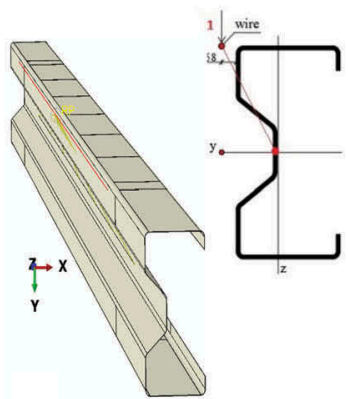

d)

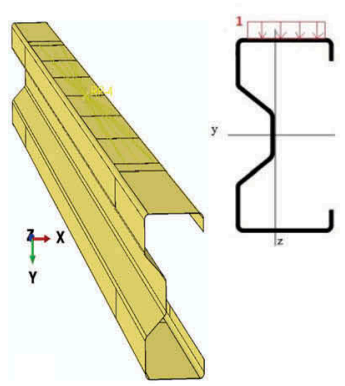

Figure 2. Load application in numerical model. a) WIRE line location, case: b) V1, c) V2, d) V3. 


\section{RESULTS OF NUMERICAL EXAMPLE}

Using Eq. 1 for simply supported, subjected to uniformly distributed load beam with the span of $2.20 \mathrm{~m}$ and made of $\Sigma 140 \times 2.5$ profile the critical moment was determined and it was at a value of $3272 \mathrm{kN} \mathrm{cm}$. The calculations were carried out for simplification of the sigma cross-section geometry in terms of omitting rounding. It is worth noting that the value of the critical bending moment calculated on the basis of the Vlasov beam theory does not take into account the loss of local stability or contour deformation. While, these phenomena can be analysed in the FEM shell model. For all developed FEM numerical models, a multimodal buckling analysis in Abaqus program was carried out. Eigenvalues and shape of three buckling modes are presented in Table 1.

Table 1. Shape of buckling mode and eigenvalue for three buckling mode.

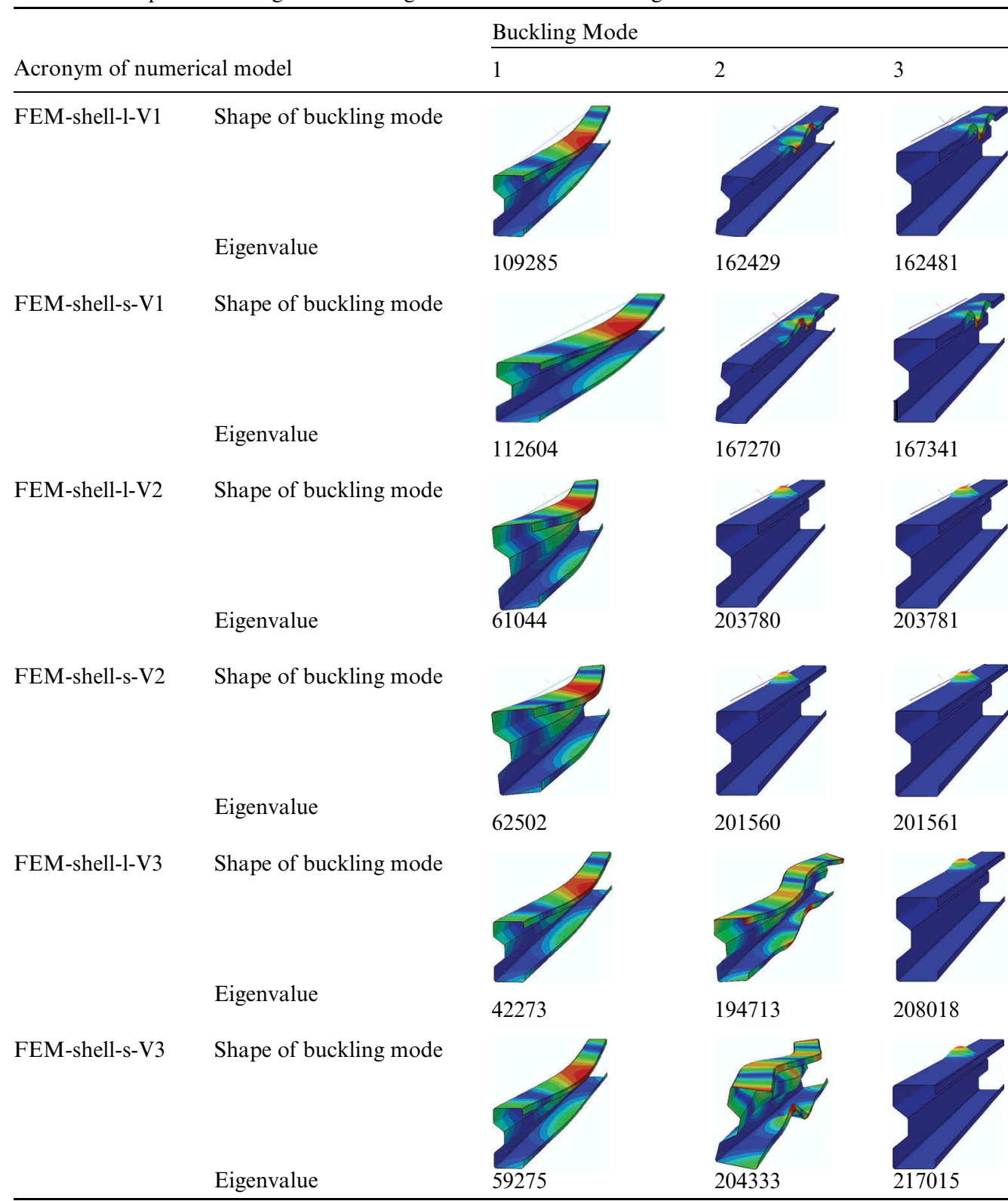


Table 2. Critical moment and critical stress for different numerical descriptions.

\begin{tabular}{lll}
\hline & Critical Moment $M_{c \mathrm{r}}$ & Critical stress $\sigma_{c r}$ \\
\cline { 2 - 3 } Acronym of numerical model & $\mathrm{kNcm}$ & $\mathrm{kN} / \mathrm{cm}^{2}$ \\
\hline FEM-shell-1-V1 & 3005 & 87.57 \\
FEM-shell-s-V1 & 3097 & 90.25 \\
FEM-shell-1-V2 & 1679 & 48.93 \\
FEM-shell-s-V2 & 1719 & 50.09 \\
FEM-shell-l-V3 & 1162 & 33.86 \\
FEM-shell-s-V3 & 1630 & 47.50 \\
Vlasov theory & 3272 & 95.35 \\
BEM-out & - & 28.92 \\
BEM-int & - & 97.62 \\
\hline
\end{tabular}

It can be clearly stated that the type of FE strongly influences on critical bending moment. The analysis of the buckling mode shape leads also to the conclusion that in all the analysed cases and for all buckling modes, the decisive instability type is local buckling of compressed flange. Therefore, it was considered justified to determine the critical stresses for the separated plate corresponding to the compressed flange using the Boundary Element Method (Eq. 4). Due to a number of simplifications related to BEM, the stability of the upper flange was analysed assuming two types of boundary condition, specifically cantilever, thus the flange was analysed as outstand compression walls (BEM-out) and simply supported where the flange was consider as internal compression (BEM-int). In order to performed comparison between analytical and FEM or BEM solution critical stresses were calculated for all considered numerical descriptions (Table 2). One can notice that the lowest values of the critical stresses are obtained for the BEM-out model, which gives the most restrictive values and, according to it, the loss of stability occurred the fastest. At the same time, the highest values of critical stresses are obtained for the BEM-int model, which means that designing the structure using this model may be dangerous. The same is the case of the critical stresses calculated on the basis of the Vlasov theory and in the case of FEM-shell-1-V1 and FEM-shell-s-V1 models. Therefore, these models should be regarded as overestimate and unreliable.

\section{CONCLUDING REMARKS}

On the basis of the conducted analyses, it is possible to formulate a number of interesting conclusions concerning the stability analysis of cold-formed steel elements.

First of all, it is clear that the type of application of the description of the calculation model strongly influences the value of critical stresses. Buckling analysis in FEM showed that the analysed element undergoes both local and global instability, and in many cases the decisive form of buckling is the local stability loss of compression flange. Unfortunately, as is known, theoretical critical bending moment obtained from analytical close form formulas based on so called Vlasov beam theory dedicated to the thin-walled elements does not take local buckling into account. Therefore, it can be seen that the load value obtained from Vlasow theory is much higher than that obtained from the FEM shell model. This means that Vlasow theory should not be directly applied in the analyzed case. It was also demonstrated that for local buckling, it is sufficient to analyse the single wall of the cross-section with the greatest plate slenderness. In this work, due to some BEM method simplification the compressed flange was consider only as outstand or internal wall, while in reality in fact there are semi-rigid support condition. That's why only the upper and lower estimates of the critical stresses were obtained. two such an analysis was performed for a compressed flange treated as a cantilever wall using BEM. On the other hand it should be emphasized that numerical FEM-shell-1-V3 and 
FEM-shell-s-V3 models which reflect the real engineering conditions of external load application simultaneously enable multimodal buckling analysis taking into account local and global buckling as well and provide reasonable values of critical stresses and thus should be regarded as the most suitable.

\section{ACKNOWLEDGEMENT}

This paper was financially supported by Lublin University of Technology: FN15/ILT/2020 and Poznan University of Technology: 0412/SBAD/0044.

\section{REFERENCES}

American Iron and Steel Institute. 1996. Specification for the Design of Cold-Formed Steel Structural.

Bródka J., Broniewicz M., Giżejowski M. 2006. Cold-formed profiles. Designer handbook. PWT, (in Polish).

Guminiak, M. 2014. An Alternative Approach of Initial Stability Analysis of Kirchhoff Plates by the Boundary Element Method. Engineering Transactions 62(1): 33-59.

Guminiak, M. 2016. The boundary element method in analysis of plates (in Polish). Poznan: Poznan Uni-versity of Technology Publishing House.

Karman T., Sechler E.E., Donnel L. H. 1932. Strength of thin plates in compression, Trans. ASME, 54.

Members. Publication CF 96-1. Washington D.C.

PN-90/B-03200: Steel structures - static calculations and design, (in Polish).

Rzeszut K., Folta W., Garstecki A. 2018. Reliability of Engineering Methods of Assessment the Critical Buckling Load of Steel Beams, [w]: Proceedings of the 22nd International Conference on Computer Methods in Mechanics, AIP Publishing, s. 150003-1-150003-7.

Rzeszut K., Szewczak I. 2017. Experimental Studies of Sigma Thin-Walled Beams Strengthen by CFRP Tapes. International Journal of Civil, Environmental, Structural, Construction and Architectural Engineering, Vol.11/2017, No.7, p.888-895. ISNI: 0000000091950263

Rzeszut K., Szewczak I., Różyło P. 2018. Issues of thin-walled sigma beams strengthened by CFRP tape in context of experimental and numerical study. Engineering Transaction, vol. 66, no. 1, 79-91.

Szczerba R. 2013. Load capacity and stability of steel C-section beams, Budownictwo i Architektura 12 (2) 283-290. (in Polish).

Zienkiewicz O.C. 1977. The finite element method in engineering science. London. McGraw Hill. 